\title{
Imaging and Interpretation of Axial Spondylarthritis: The Radiologist's Perspective-Consensus of the Arthritis Subcommittee of the ESSR
}

\author{
Claudia Schueller-Weidekamm, MD, PhD, MBA ${ }^{1}$ Vasco V. Mascarenhas, MD, MSc, EMBA ${ }^{2}$ \\ Iwona Sudol-Szopinska, MD, $\mathrm{PhD}^{3}$ Nathalie Boutry, MD, $\mathrm{PhD}^{4}$ Athena Plagou, MD, $\mathrm{PhD}^{5}$ \\ Andrea Klauser, MD ${ }^{6}$ Marius Wick, $\mathrm{MD}^{7}$ Hannes Platzgummer, MD ${ }^{1}$ Lennart Jans, MD, $\mathrm{PhD}^{8}$ \\ Adam Mester, MD, $\mathrm{PhD}^{9}$ Franz Kainberger, MD, $\mathrm{PhD}^{1}$ Gunnar Aström, MD, $\mathrm{PhD}^{10}$ \\ Giuseppe Guglielmi, MD ${ }^{11}$ Iris Eshed, MD ${ }^{12}$
}

\footnotetext{
${ }^{1}$ Division of Neuroradiology and Musculoskeletal Radiology, Department of Biomedical Imaging und Image-guided Therapy, Medical University of Vienna/Vienna General Hospital, Vienna, Austria

${ }^{2}$ Hospital da Luz, ESS, Lisbon, Portugal

3 Department of Radiology, Institute of Rheumatology, Warsaw and Department of Diagnostic Imaging, Warsaw Medical University, Warsaw, Poland

${ }^{4}$ Department of Musculoskeletal Radiology, Centre Hospitalier Universitaire de Lille and Hôpital Roger Salengro, Lille, France

${ }^{5}$ Radiologist, Private Institute, Athens, Greece

${ }^{6}$ Department of Radiology, Innsbruck Medical University, Section Rheumatology and Sports Imaging, Innsbruck, Austria

7 Department of Radiology, Karolinska University Hospital, Stockholm, Sweden

8 Department of Radiology, Ghent University Hospital, Ghent, Beligium

${ }^{9}$ Department of Radiology, National Institute of Rheumatology and Physiotherapy, Budapest, Hungary

${ }^{10}$ Department of Radiology, Oncology and Radiation Sciences, Section of Radiology, Uppsala University, Uppsala, Sweden

${ }^{11}$ Department of Radiology, University of Foggia, Foggia, Italy

12 Department of Diagnostic Imaging, Sheba Medical Center, Sackler School of Medical, Tel Aviv, Israel
}
Address for correspondence Claudia Schueller-Weidekamm, MD, $\mathrm{PhD}, \mathrm{MBA}$, Division of Neuroradiology and Musculoskeletal Radiology, Department of Biomedical Imaging und Image-guided Therapy, Medical University of Vienna/Vienna General Hospital, Waehringer Guertel 18-20, A-1090 Vienna, Austria
(e-mail: claudia.schueller-weidekamm@meduniwien.ac.at).

Semin Musculoskelet Radiol 2014;18:265-279.

\begin{abstract}
This article reflects the radiologist's perspective on the imaging and interpretation of axial spondylarthritis (SpA). The arthritis subcommittee of the European Society of Skeletal Radiology provides a consensus for the following questions: When and how should we image? How should we analyze the images? How should we interpret the imaging findings? To answer these questions, we address the indications in imaging

Keywords

- spondylarthritis

- magnetic resonance imaging

- sacroiliitis

- diagnosis axial SpA and the different imaging techniques, with a special focus on magnetic resonance imaging protocols. The value of different imaging modalities is discussed. For adequate image analysis, knowledge of the anatomy and the pathologic changes in chronic and acute inflammation of the sacroiliac joints and the spine is mandatory. Differential diagnoses of inflammatory lesions of the sacroiliac joints and the spine are addressed due to their importance in image interpretation.
\end{abstract}

Issue Theme Spine; Guest Editor, Mara Epermane, MD
Copyright @ 2014 by Thieme Medical Publishers, Inc., 333 Seventh Avenue, New York, NY 10001, USA. Tel: +1(212) 584-4662.
DOI http://dx.doi.org/ 10.1055/s-0034-1375569. ISSN 1089-7860. 
Seronegative axial spondylarthritis (SpA) is a chronic inflammatory disease that affects the sacroiliac joints (SIJs) and the spine. On average, axial SpA is misdiagnosed for up to 7 years from the onset of symptoms that consist mainly of inflammatory back pain (IBP) for at least 3 months and restricted spinal mobility. Magnetic resonance imaging (MRI) of the SIJs has increased dramatically during the last decade to enable the early diagnosis of axial SpA and facilitate early treatment, thus preventing structural damage. The earlier adequate therapy is initiated, the better the patient's outcome. ${ }^{1}$ Debate continues about the appropriate imaging in patients with suspected or confirmed SpA. Questions about the usefulness of (dynamic) contrast-enhanced sequences in MRI and of imaging of the whole spine versus the lumbar spine still remain unanswered among radiologists and rheumatologists. The anatomy and histology of the SIJs are important for planning the MRI examination and for image interpretation. A thorough knowledge of normal imaging findings, typical features in SpA, and the differential diagnosis are crucial in image analysis and interpretation. The Subcommittee on Arthritis Imaging of the European Society of Skeletal Radiology (ESSR) has established the following recommendations for imaging axial SpA. The recommendations are a consensus of the subcommittee and based on expert opinion and on the literature.

\section{When Should We Image?}

\section{Indication for Imaging}

Axial SpA can be distinguished on the basis of patient history and typical clinical and laboratory findings that were first described by Calin et al. $^{2}$ Patients who experience chronic (nonradicular) $\mathrm{IBP}^{3}$ with an insidious onset of symptoms $<45$ years of age and a duration of at least 3 months should be initially imaged with radiography, according to the Assessment of SpondyloArthritis (ASAS) criteria. ${ }^{4}$ We should be aware that the diagnosis of SpA is delayed for 5 to 7 years after the onset of symptoms if only radiographs are obtained. ${ }^{5} \mathrm{~A}$ negative examination of the pelvis or spine bears the risk of a delayed diagnosis because radiographs cannot exclude the possibility of SpA. This results in our recommendation that, in case of negative radiographs in patients with a suspicion of $\mathrm{SpA}$, MRI is mandatory to look for early inflammatory lesions.

Both the clinical and the imaging arm are considered equally important for the classification criteria of ASAS., ${ }^{4,6}$ In combination with at least one other feature of $\mathrm{SpA}$, sacroiliitis, either diagnosed by radiographs (radiographic stage) or diagnosed by MRI (nonradiographic stage), is referred to as the imaging arm. HLA-B27-positivity, in combination with at least two other features of SpA, is referred to as the clinical arm. This underlines the importance of imaging.

\section{Value of Different Imaging Modalities}

\section{Radiography}

Radiography is useful as a baseline imaging technique to document the presence and progression of structural changes, such as sclerosis, erosions, and osteoproliferations
Table 1 Modified New York criteria for the classification of ankylosing spondylitis ${ }^{8}$

\begin{tabular}{l} 
Low back pain for at least 3 months improved by exercise \\
and not relieved by rest \\
\hline Limitation of lumbar spine in sagittal and frontal planes \\
\hline $\begin{array}{l}\text { Reduced chest expansion relative to normal values for } \\
\text { age and sex }\end{array}$ \\
\hline Bilateral sacroiliitis grades $2-4$ \\
\hline Unilateral sacroiliitis grades $3-4$ \\
\hline
\end{tabular}

Note: According to the modified New York criteria, the diagnosis of ankylosing spondylitis is definite if radiographic sacroiliitis is present with at least one of the clinical symptoms.

of the pelvis or spine, and to rule out complications in SpA such as fractures or other causes of low back pain such as tumors, infections, and acute or chronic trauma. However, we should be aware of the low sensitivity of radiographs for the detection of early SpA. ${ }^{7}$ High inter- and intraobserver disagreements in the analysis of radiographs might be caused by the two-dimensional view of the complex anatomy of the SIJs. Care should be taken to analyze the shape and height of the vertebral bodies to identify osteoporotic fractures, which are one of the complications in SpA.

The ESSR subcommittee agrees that radiographs of the pelvis should be evaluated according to the modified New York criteria for ankylosing spondylitis (AS). These criteria are fulfilled if, in addition to one clinical criteria, bilateral sacroiliitis grade 2 or unilateral sacroiliitis grade 3 to 4 on radiographs are present (-Tables $\mathbf{1}$ and 2). ${ }^{8}$ Again, it should be noted that the modified New York criteria are not useful for the diagnosis of early SpA because the preradiographic stage cannot be detected. Radiographic evidence of inflammation reflects a structural change, which is usually a sign of advanced disease.

\section{Magnetic Resonance Imaging}

MRI is the imaging modality of choice for the detection of early inflammatory spinal and sacroiliac lesions in SpA. Inflammatory changes in the SIJs and the spine can be detected by MRI before they can be seen by radiographs or computed tomography (CT). Therefore, MRI is established as the most sensitive imaging modality for the early detection of axial SpA. ${ }^{9}$ The high soft tissue and bone marrow contrast is

Table 2 Scoring of radiographic findings on radiographs of the pelvis for the New York criteria ${ }^{8}$

\begin{tabular}{|l|l|}
\hline Grade & Radiographic findings \\
\hline 0 & Abnormalities are present \\
\hline 1 & Suspicious areas are noted \\
\hline 2 & Minor erosions \\
\hline 3 & Unequivocal abnormalities in the joint \\
\hline 4 & Severe change in the joint \\
\hline
\end{tabular}



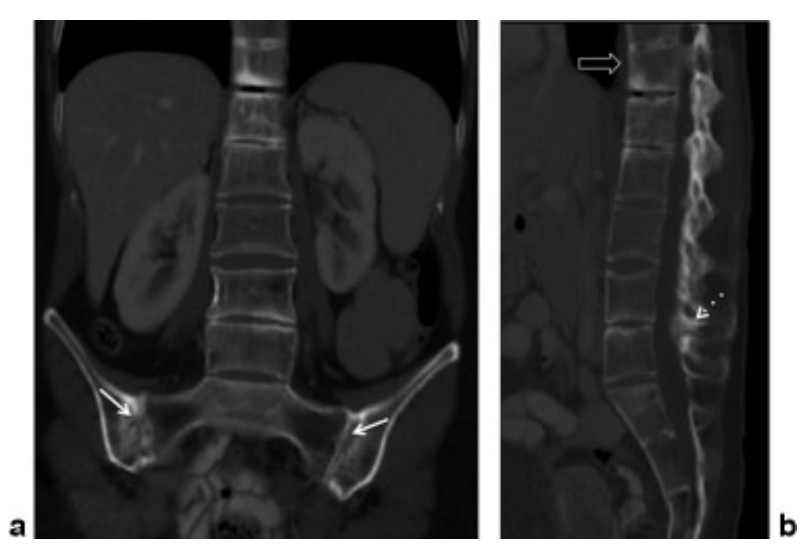

Fig. 1 Computed tomography examination in this 38-year-old woman was performed due to chronic inflammatory bowel disease. (a) Coronal and (b) sagittal reformation in the bone window show ankylosis of the sacroiliac joints (arrows), wedged shaped T12 due to insufficiency vertebral fracture (open arrow), sclerosis of the anterior vertebral corners, erosions of the vertebral end plates, and ankylosis of the lumbar facet joints (dashed arrow).

beneficial for the detection of bone marrow edema (BME), with T2-weighted fat-suppressed sequences that enable the diagnosis of active sacroiliitis in the preradiographic stage. According to the ASAS criteria, sacroiliitis, defined by subchondral/periarticular BME or osteitis on contrast-enhanced MRI, is mandatory for a "positive" MRI in SpA. In the absence of BME, capsulitis, synovitis, and enthesitis do not indicate SpA. Subchondral/periarticular fat infiltration and sclerosis seen on T1-weighted images (with or without fat suppression) might indicate areas of previous inflammation and is common in SpA. ${ }^{10}$ MRI enables confirmation of a diagnosis of early SpA, suspected on the basis of clinical aspects, as early as 4 months after symptom onset. ${ }^{11}$ However, according to the ASAS SpA definition, a negative MRI cannot rule out SpA if HLA-B27 positivity and at least two other features of SpA are present. The low specificity (0.88) of MRI in the diagnosis of SpA according to the ASAS criteria might be explained by the subchondral/periarticular BME that also occurs in asymptomatic individuals and in patients with mechanical back pain in the SIJ. ${ }^{12}$ The combined scanning of the whole spine and the
SIJs enhances confidence in diagnosing SpA compared with SIJ MRI alone in patients with nonradiographic axial SpA. ${ }^{13}$ Diffusion-weighted MRI and whole-body MRI have been used in a few reports, however, its clinical or research potential is not yet established.

MRI plays a distinguished role in imaging the response to treatment and complications, or in ruling out differential diagnoses that can potentially cause lower back pain. The prognostic role of MRI is currently under debate. For spinal involvement, BME in the vertebral corners might be a sign of active inflammation, and it may evolve into new syndesmophytes and erosions. ${ }^{14}$

\section{Computed Tomography}

CT directly visualizes structural changes such as erosions, subchondral/periarticular sclerosis in the SIJ, bony proliferations, or ankylosis of the joint space at high spatial resolution, and therefore it provides a higher sensitivity for the detection of structural changes, compared with radiography. The three-dimensional (3D) data set of the CT examination enables multiplanar and 3D reformation of the SIJ space and the vertebral column ( $\mathbf{- F i g . 1}$ 1a, b). Radiation exposure from the CT examination, primarily in young patients, is one eminent argument against the routine recommendation of CT examination of the SIJs or spine. Therapeutic decisions are rarely influenced by structural changes detected on CT. Positron emission tomography (PET)/CT might play a role in the future because the measured fluorodeoxyglucose uptake is a biomarker for inflammation, and additional structural changes can be nicely depicted. Despite these advantages, radiologists are responsible for the radiation exposure and should carefully consider the indications for $\mathrm{PET} / \mathrm{CT}$.

\section{Ultrasound}

To date, only a few studies have reported the role of ultrasound (US) in the evaluation of the SIJs. ${ }^{15}$ It is debatable whether it is possible to image synovitis and effusion of the SIJ space. The deeper, more anteriorly located part of the SIJ is not visible on US. For these reasons, we do not recommend relying solely on US findings for the diagnosis of axial SpA

Table 3 MRI protocol for total spine at $3 \mathrm{~T}$

\begin{tabular}{|l|l|l|l|l|l|l|l|l|l|}
\hline Sequence & Plane & TR & TE & IR & FOV & Matrix & ST & Gap & Time \\
\hline STIR & Sagittal & 4266 & 75 & 220 & $380 \times 380$ & $424 \times 300$ & $3 \mathrm{~mm}$ & 0.3 & $5: 58$ \\
\hline T1 TSE & Sagittal & 447 & 8.2 & & $380 \times 380$ & $424 \times 304$ & $3 \mathrm{~mm}$ & 0.4 & $4: 41$ \\
\hline
\end{tabular}

TE, TR, and inversion time are given in milliseconds. Scanning time is given in minutes. Slice thickness, field of view, and gap are given in mm. Abbreviations: FOV, field of view; IR, inversion recovery; ST, slice thickness; STIR, short tau inversion recovery; TSE, turbo spin echo.

Table $4 \mathrm{MRI}$ protocol for total spine at $1.5 \mathrm{~T}$

\begin{tabular}{|l|l|l|l|l|l|l|l|l|l|}
\hline Sequence & Plane & TR & TE & IR & FOV & Matrix & ST & Gap & Time \\
\hline STIR & Sagittal & 5000 & 108 & 140 & $350 \times 350$ & $288 \times 384$ & $3 \mathrm{~mm}$ & 0.3 & $2: 45$ \\
\hline T1 TSE & Sagittal & 846 & 10 & & $350 \times 350$ & $384 \times 384$ & $3 \mathrm{~mm}$ & 0.3 & $5: 02$ \\
\hline
\end{tabular}

TE, TR, and inversion time are given in milliseconds. Scanning time is given in minutes. Slice thickness, field of view, and gap are given in mm. Abbreviations: FOV, field of view; IR, inversion recovery; ST, slice thickness; STIR, short tau inversion recovery; TSE, turbo spin echo. 


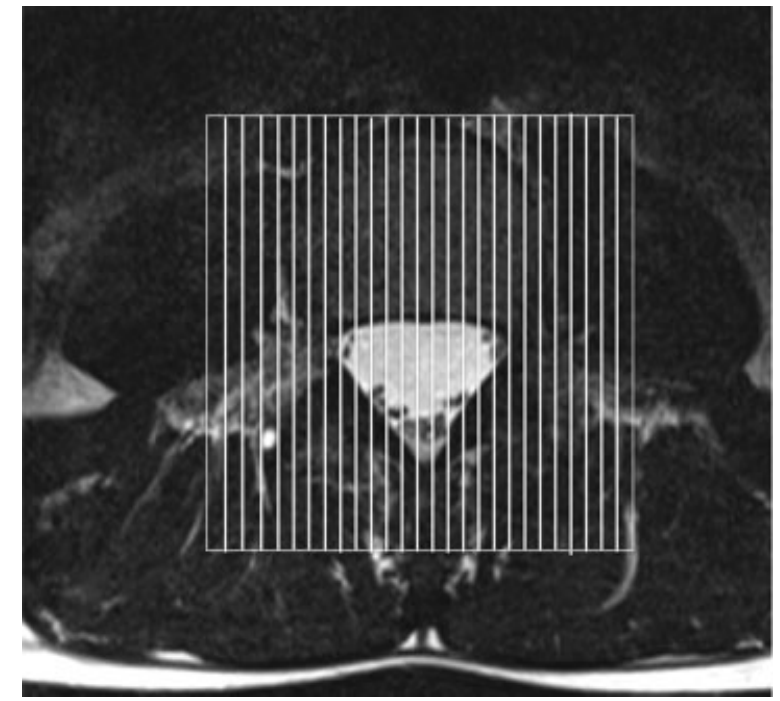

Fig. 2 The number of slices in the sagittal plane should be increased to extend the scanning range and to image the lateral paravertebral segments. The white stripes correspond to the single slices in the sagittal plane.

because US cannot replace MRI or radiography for the diagnosis of SpA. Nonetheless, there is an established role for US in minimally invasive US-guided therapy for infiltration of the SIJs and in the assessment of peripheral involvement in SpA. Inflammation in the supraspinal ligaments can be detected noninvasively with Doppler US.

\section{Dual-Energy X-ray Absorptiometry}

Patients with SpA are at higher risk for spinal fractures. We agree that dual-energy X-ray absorptiometry is indicated in nontraumatic SpA patients with a suspicion of spinal fracture to measure the bone mineral density and calculate the increased risk of fractures.

\section{How Should We Image?}

\section{Technical Aspects of Radiography}

The first-line imaging modality is radiography. We recommend imaging the whole spine anteroposteriorly and laterally.

The SIJ should be imaged on the anteroposterior view of the pelvis in the erect position. The frequent involvement of the hips in axial SpA justifies imaging of the pelvis. We do not recommend additional SIJ oblique views due to unnecessary radiation exposure and lack of additional diagnostic value.

\section{Technical Aspects of MRI}

Patients should be scanned in the supine position. According to previous studies, the total spine should be imaged because $>50 \%$ of all active lesions are usually located in the thoracic spine. $^{16}$

Mandatory MRI sequences for the spine and SIJs in adults and children are provided in -Tables 3-6. The provided protocols are a consensus of the arthritis subcommittee of the ESSR. - Table 3 shows the MRI protocol for the total spine at 3T; - Table 4 shows the MRI protocol for the total spine at $1.5 \mathrm{~T}$. The scanning range for the spine should include the lateral paravertebral segments because the costovertebral and costotransversal joints, respectively, should be subject to investigation (-Fig. 2). For the SIJs, the MRI protocol 3T is provided in - Table 5. - Table 6 shows the MRI protocol for SIJs at $1.5 \mathrm{~T}$. In doubtful cases, we recommend additional

Table 5 MRI protocol for sacroiliac joints at $3 \mathrm{~T}$

\begin{tabular}{|l|l|l|l|l|l|l|l|l|l|}
\hline Sequence & Plane & TR & TE & IR & FOV & Matrix & ST & Gap & Time \\
\hline STIR & Paracoronal & 4263 & 75 & 220 & $380 \times 380$ & $424 \times 300$ & $3 \mathrm{~mm}$ & 1 & $6: 00$ \\
\hline T1 TSE & Paracoronal & 625 & 10 & & $230 \times 230$ & $576 \times 382$ & $3 \mathrm{~mm}$ & 0.4 & $4: 00$ \\
\hline PD FS & Para-axial & 8140 & 30 & & $350 \times 254$ & $576 \vee 382$ & $3 \mathrm{~mm}$ & 0.4 & $4: 30$ \\
\hline
\end{tabular}

Abbreviations: FOV, field of view (mm); FS, fat suppression; IR, inversion recovery; PD, proton density; ST, slice thickness; STIR, short tau inversion recovery; TE, echo time (ms); TR, repetition time (ms); TSE, turbo spin echo.

Table 6 MRI protocol for sacroiliac joints at $1.5 \mathrm{~T}$

\begin{tabular}{|l|l|l|l|l|l|l|l|l|l|}
\hline Sequence & Plane & TR & TE & IR & FOV & Matrix & ST & Gap & Time \\
\hline STIR & Paracoronal & 5030 & 67 & 150 & $320 \times 320$ & $320 \times 320$ & $3 \mathrm{~mm}$ & 0.6 & $5: 59$ \\
\hline T1 TSE & Paracoronal & 595 & 20 & & $320 \times 320$ & $384 \times 512$ & $3 \mathrm{~mm}$ & 0.6 & $4: 38$ \\
\hline
\end{tabular}

Abbreviations: FOV, field of view (mm); IR, inversion recovery; ST, slice thickness; STIR, short tau inversion recovery; TE, echo time (ms); TR, repetition time (ms); TSE, turbo spin echo.

Table 7 Contrast-enhanced MRI sequences for sacroiliac joints at 3T in doubtful cases ${ }^{\mathrm{a}}$

\begin{tabular}{|c|c|c|c|c|c|c|c|c|}
\hline Sequence & Plane & TR & TE & FOV & Matrix & ST & Gap & Time \\
\hline T1 TSE FS & Para-axial & 690 & 8 & $250 \times 250$ & $276 \times 236$ & $3 \mathrm{~mm}$ & 0.4 & $4: 00$ \\
\hline T1 TSE FS & Paracoronal & 597 & 10 & $260 \times 260$ & $322 \times 284$ & $3 \mathrm{~mm}$ & 0.4 & $7: 00$ \\
\hline
\end{tabular}

Abbreviations: FOV, field of view (mm); FS, fat suppression; ST, slice thickness; TE, echo time (ms); TR, repetition time (ms); TSE, turbo spin echo. ${ }^{\mathrm{a}}$ After intravenous contrast medium (gadolinium) with fat saturation). 

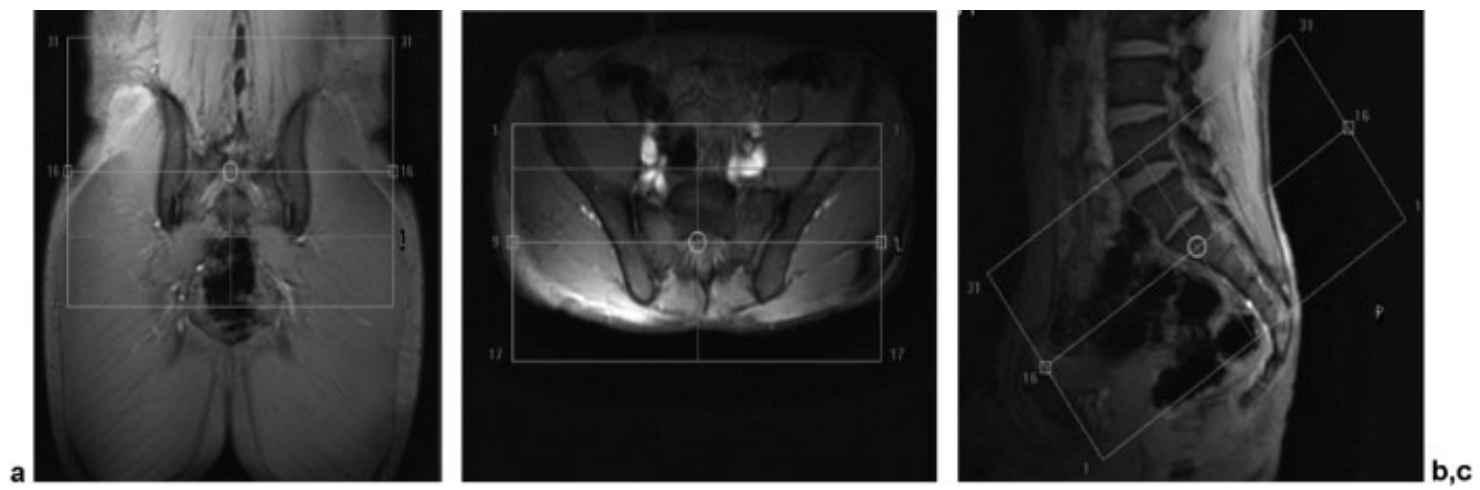

Fig. 3a-c Three-dimensional scout in (a) coronal, (b) axial, and (c) sagittal plane for planning of the MR sequence in the para-axial plane. The paraaxial plane should be parallel to the upper vertebral end plate of the S1 vertebra.

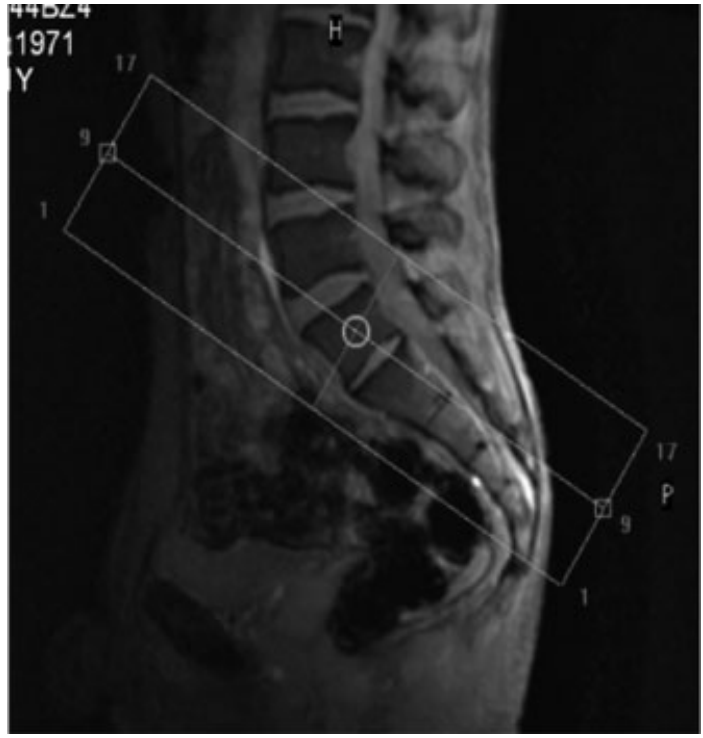

Fig. 4 The paracoronal plane should be parallel to the long axis of the sacrum. sequences for the SIJs to evaluate the differential diagnosis (-Table 7; - Figs. 3a-c and 4a-c). A slice thickness of $3 \mathrm{~mm}$ should not be exceeded to guarantee appropriate depiction of erosions or corner inflammatory lesions.

A specific distinction between the two joint portions by MR imaging is precisely seen on a para-axial slice orientation. The para-axial plane is required to evaluate the exact anatomical location of the pathologies and to avoid pitfalls that might occur on paracoronal planes due to partial volume effects. The para-axial plane enables the detection of the strong interosseous ligaments superiorly and posteriorly that form the syndesmosis, and to differentiate the two cartilage layers of the inferiorly and anteriorly cartilaginous-covered articulation. Posteriorly located small vessels in the middle and distal third and interosseous ligaments should not be mistaken as synovitis or erosions, respectively. Posterior iliac clefts should not be mistaken as erosions if only paracoronal slice orientation is performed. A synovial recess has been described inferiorly on the iliac side. ${ }^{17}$ Both hip joints should be included in the scanning range in the para-axial plane.
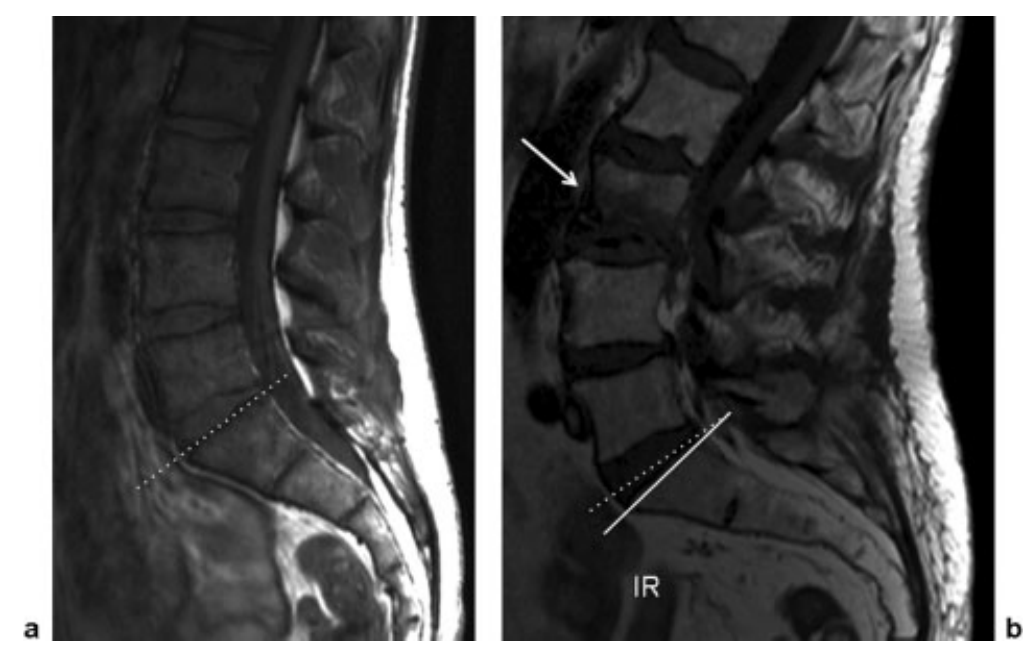

Fig. 5 The shape of the male pelvis differs from the female pelvis. The male pelvis (a) is more erect and the end plate of S1 is angled more horizontally (dashed line) compared with S1 (white line) in the more convex-shaped female pelvis (b). The bone marrow signal on the T1-weighted sequence differs in these two patients. In (a), the 24-year-old male patient shows a higher ratio of hematopoietic bone marrow, whereas the 92-year-old woman has fat bone marrow that results in a high signal on T1. Osteoporotic fracture is seen in L3 (b, arrow). 

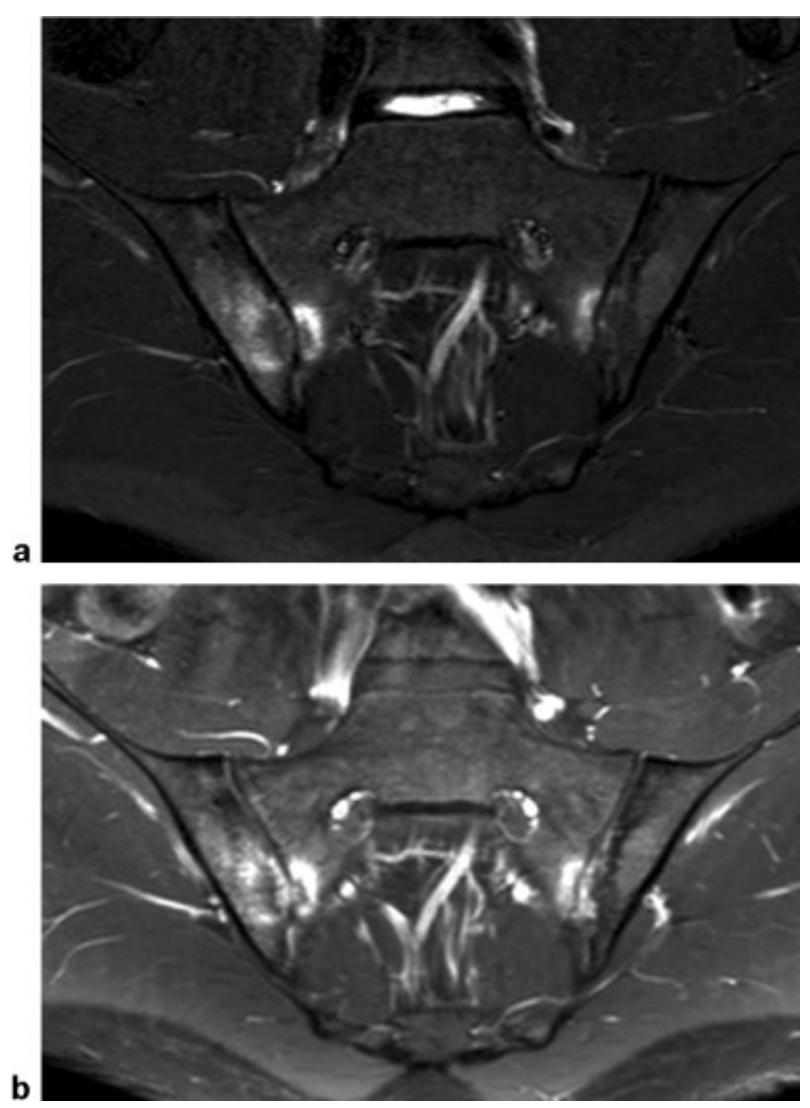

Fig. 6 Sacroiliitis in a 20-year-old male patient. Bone marrow edema is seen on both the paracoronal short tau inversion recovery (a) and contrastenhanced T1-weighted fat-suppressed (b) sequence. In this case, additional information could not be obtained after contrast medium.
The paracoronal orientation is superior for visualization of the subchondral osseous areas.

Due to the anatomical differences between the male and female pelvis, paracoronal and para-axial planes are performed at different angulations ( - Fig. 5a, b).

Should Contrast Medium Be Included in the MRI Protocol? Currently, it is under debate whether the application of contrast media provides an additional benefit with regard to diagnostic value in MRI for the detection of sacroiliitis, capsulitis, enthesitis, and synovitis. ${ }^{18}$ In case of BME, which is the main and mandatory feature in the diagnosis of axial SpA, short tau inversion recovery (STIR) or fat-suppressed T2weighted sequences are sufficient ( $\mathbf{- F i g . 6 a , b )}$. The radiologists of the ESSR strongly believe that contrast medium definitely is of diagnostic importance for the differentiation of diagnoses other than sacroiliitis and should be applied in doubtful cases ( $\mathbf{- F i g}$. 7a, b). In children, the use of contrast medium is generally accepted for the detection of early subtle inflammatory changes in juvenile SpA. ${ }^{19}$

\section{Image Interpretation Pearls}

\section{Sacroiliac Joints}

Sacroiliac joints are the most frequently involved site in SpA. According to histology findings, in SIJs, the early lesions are depicted as tiny discontinuities of the subchondral bone plate along with subcortical edema, typically located at the inferior half of the joints and at the iliac surface, which is lined by fibrocartilage and less resistant. BME, the most important finding in SpA, is considered an active lesion, as well as
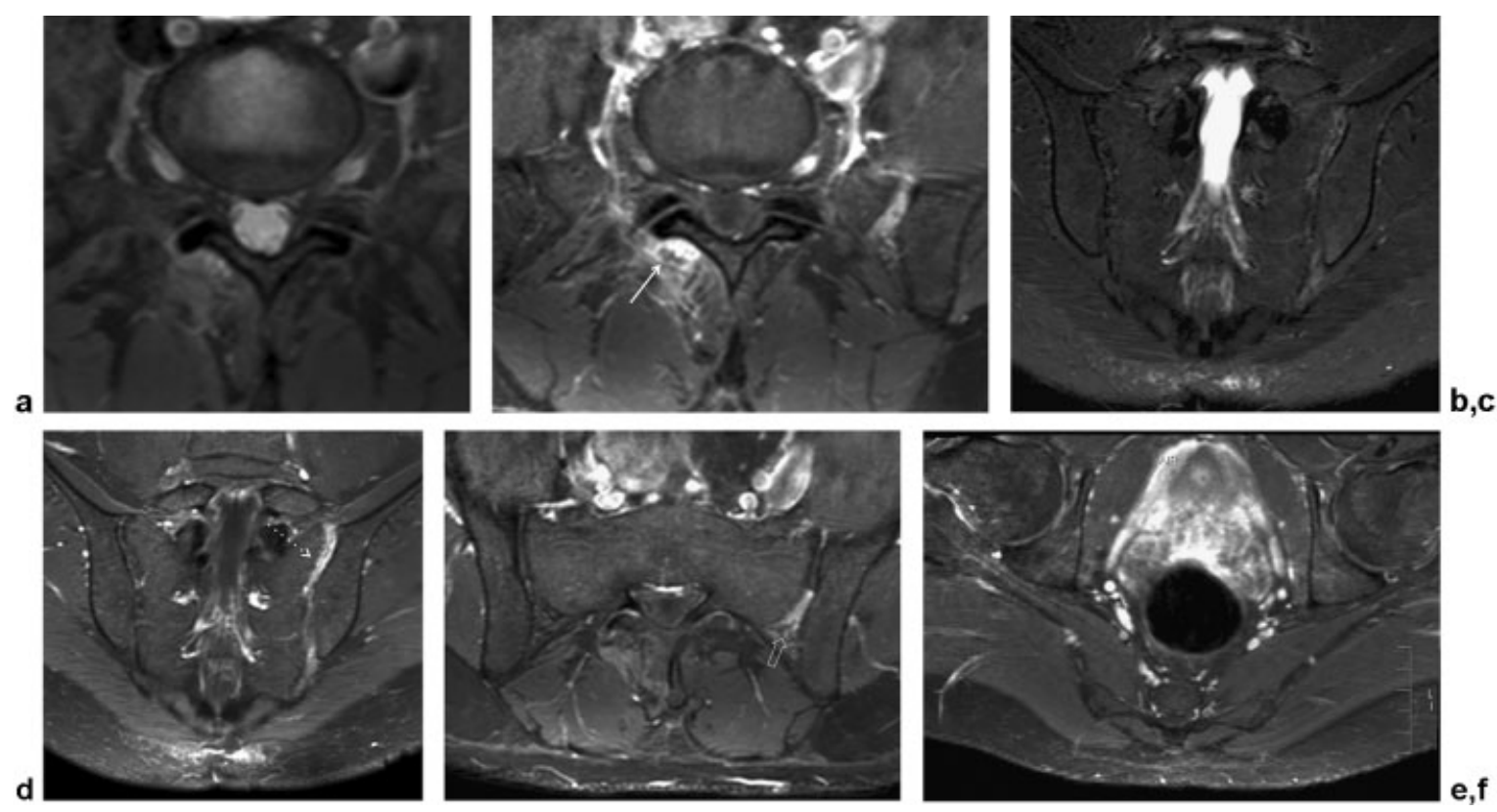

Fig. 7 (a-e) The benefit of contrast medium is obvious in this patient. The inflammation of the joint capsule of the right facet joint (arrow) is slightly better seen on the contrast-enhanced fat-suppressed T1-weighted sequence (b), compared with the fat-suppressed proton-density-weighted sequence (a). The inflammation (dashed arrow) in the left sacroiliac joint (SI)) after contrast medium (d) was missed on the short tau inversion recovery images (c). The importance of the paraaxial plane for anatomical correlation is demonstrated on the contrast-enhanced fat-suppressed T1-weighted sequences (e). The inflammation affects the posterior interosseous ligaments (open arrow), whereas the cartilage portion is not involved. Inflammation of the hip joints can be excluded on the para-axial sequence (f). 


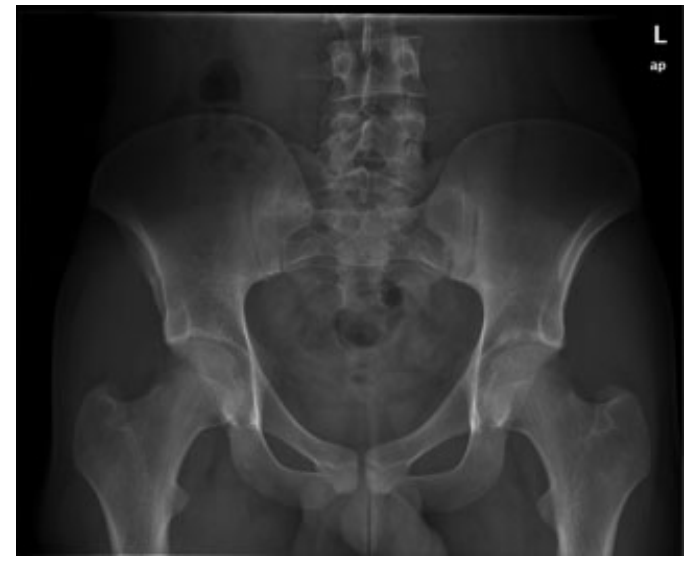

Fig. 8 Radiograph in the anteroposterior view of the pelvis of a 27year-old man. The left sacroiliac joint shows subchondral sclerosis, and irregularities of the joint space indicate small erosions. This patient was graded according to the New York criteria as grade 2 on the left side and grade 0 (no abnormalities) on the right side.

a

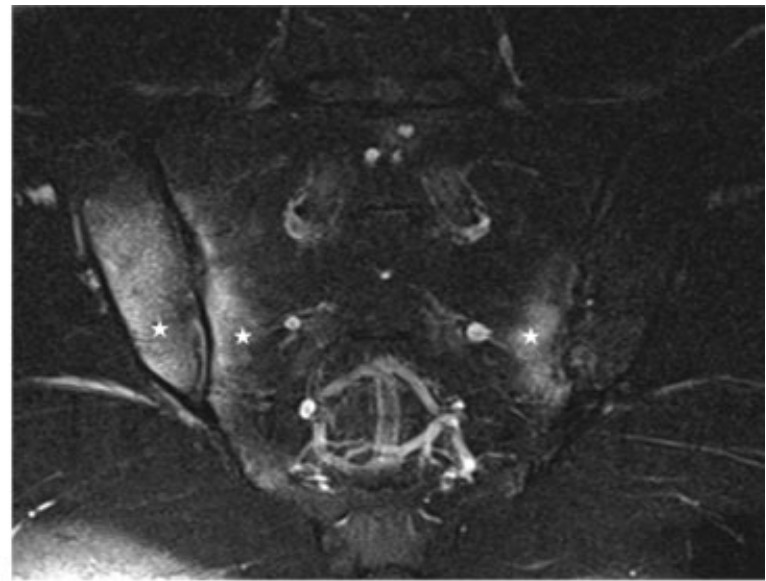

b

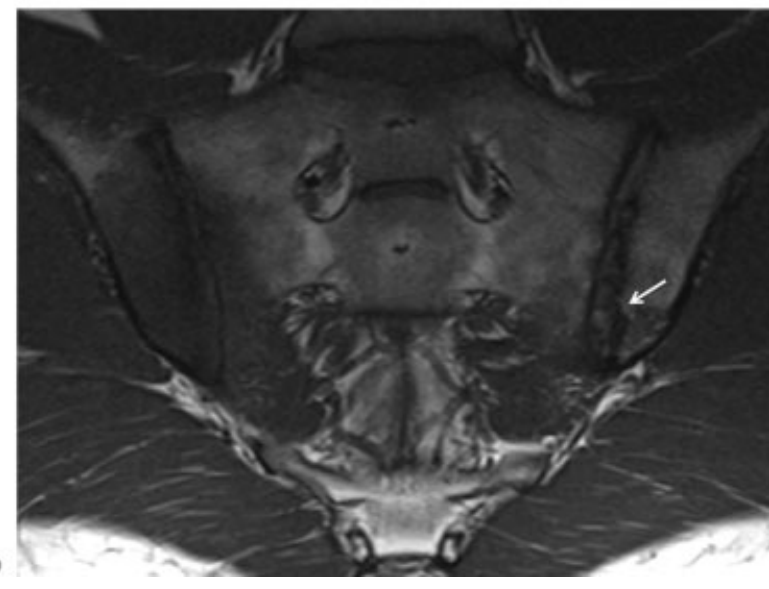

Fig. 9 Corresponding MRI of the same 27-year-old man. Short tau inversion recovery sequence (a) shows extensive bone marrow edema (BME), particularly on the sacral and ialiac side of the right SIJ (asterisks). Corresponding to the radiograph of the pelvis, the joint space is preserved without distinctive erosions. Moderate BME is seen on the sacral side of the left SIJ. The subchondral sclerosis and prominent erosions in the middle and the distal third of the left SIJ are better depicted on the T1-weighted sequence (b). Erosions result in a pseudo-dilatation of the SIJ, the so-called string-of-pearls sign (arrow).

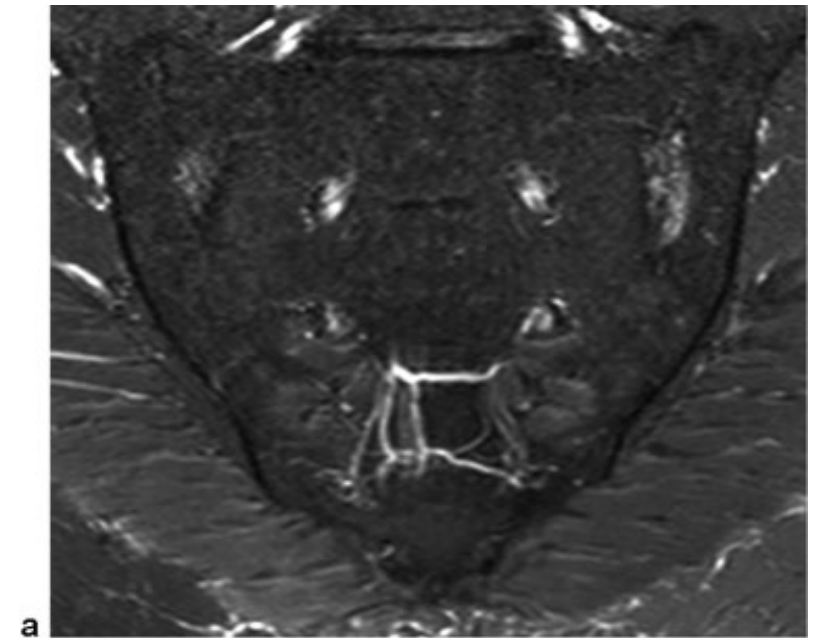
appearance), subchondral/periarticular sclerotic areas, and joint space narrowing with bony bridges, which can progress to fusion of the joint (ankylosis).

\section{Radiography}

On radiographs, SIJs should be analyzed according to the New York criteria. Structural changes, such as subchondral sclerosis, erosions, or changes of the width of the SIJ space, are characteristic imaging features (-Fig. 8).

\section{MRI}

MRI is preeminent in detecting active lesions, mainly BME in the subchondral/periarticular areas, which is of high signal intensity on STIR and T2, and of low signal intensity on T1weighted sequences. According to ASAS criteria, sacroiliitis is 


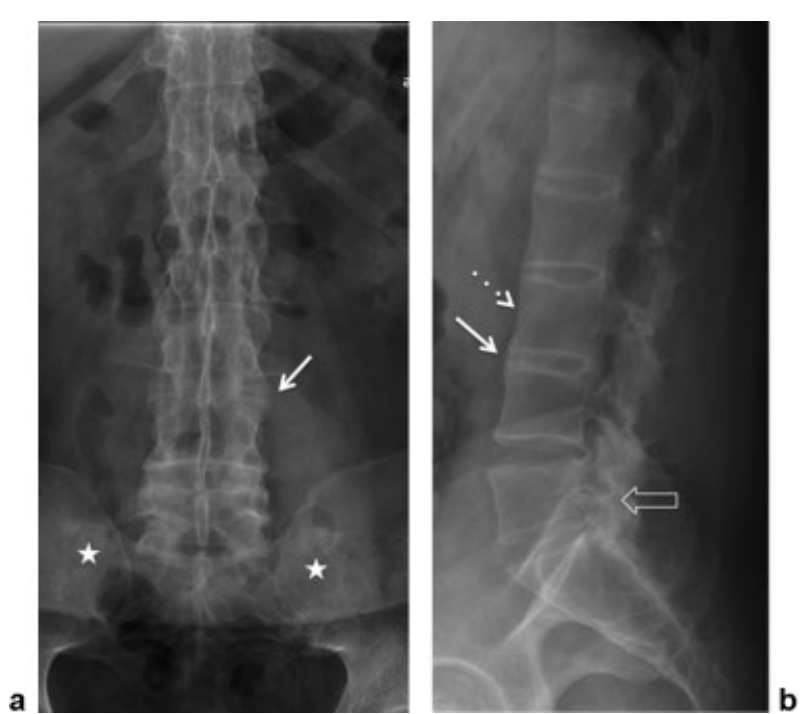

Fig. 11 Radiographs of the lumbar spine in the (a) anteroposterior and (b) lateral views of a 37-year-old man with long-standing spondylarthritis. Syndesmophytes can be depicted on both images

(arrows), and squaring of the vertebral body is seen (dashed arrow). Extensive osteoproliferations of the L5-S1 facet joints cause bilateral stenosis of the neuroforamen. Ankylosis of the facet joints is present (open arrow). Both sacroiliac joints are completely obscured on the anteroposterior view (asterisks).

defined as BME that is present on at least two consecutive slices if only one lesion is seen, or, alternatively, at least two lesions on one slice should be present. ${ }^{21}$ We recommend considering the size of areas with subchondral BME for the diagnosis of sacroiliitis ( $\mathbf{- F i g . 9 a , ~ b ) . ~ I n ~ o u r ~ o p i n i o n , ~ i n ~ t h e ~}$ absence of additional imaging features of $\mathrm{SpA}$ in the SIJs or spine, two tiny lesions $<1 \mathrm{~cm}$ in diameter are not sufficient for the diagnosis of $\mathrm{SpA}$, particularly if these lesions are located in the proximal or distal margins of the SIJs.

In chronic sacroiliitis, subchondral/periarticularly located fat replacement of the BME might occur, which is of low signal intensity on STIR and of high signal intensity on T1-and T2weighted sequences. Erosions are defined as a discontinuity or blurring of the cortical bone. Sclerosis is of low signal in all sequences. In ankylosis, bony bridges with fatty bone marrow and obscuring of the joint space are seen ( $\mathbf{- F i g . 1 0 a , b}$ ).

\section{Spine}

The spine is the second most frequent site of involvement in SpA. The subtypes of SpA show different morphological changes. In AS, the first abnormalities are erosions at the anterior aspect of the thoracolumbar vertebral bodies, ${ }^{22}$ followed by diskitis. Later, the erosions are associated with sclerotic changes and syndesmophytes, which, in long-standing disease, tend to fuse. In psoriatic arthritis, the most typical findings are parasyndesmophytes, followed by spondylitis, with BME of the entire vertebral body. In reactive arthritis, spine involvement is rare and characterized by large paravertebral ossifications.

The distribution pattern of morphological lesions including corner inflammatory lesions, central inflammatory lesions, and BME in the lateral spinal segment (lateral inflammatory lesions: pedicle, costotransversal, and costovertebral joint), is characteristic of SpA. ${ }^{23}$

\section{Radiography}

Radiographs should be evaluated for structural changes of the spine, such as proliferative bony changes or erosions of the vertebral corners. Syndesmophytes are pathognomonic in $\mathrm{SpA}$ and more vertically located compared with spondylophytes in degenerative diseases of the spine. Shiny corners represent sclerotic changes of vertebral corners and appear in later stages, as well as squaring of the vertebral body. Erosions of the vertebral end plate, usually centrally located, are the morphological correlation of destructive diskovertebral lesions (Andersson lesions). Another imaging feature in radiography is the calcification of the ligaments that results in the "bamboo spine." Ankylosis of the facet joints, costovertebral, or costotransversal joints, or even of the vertebral unit, can

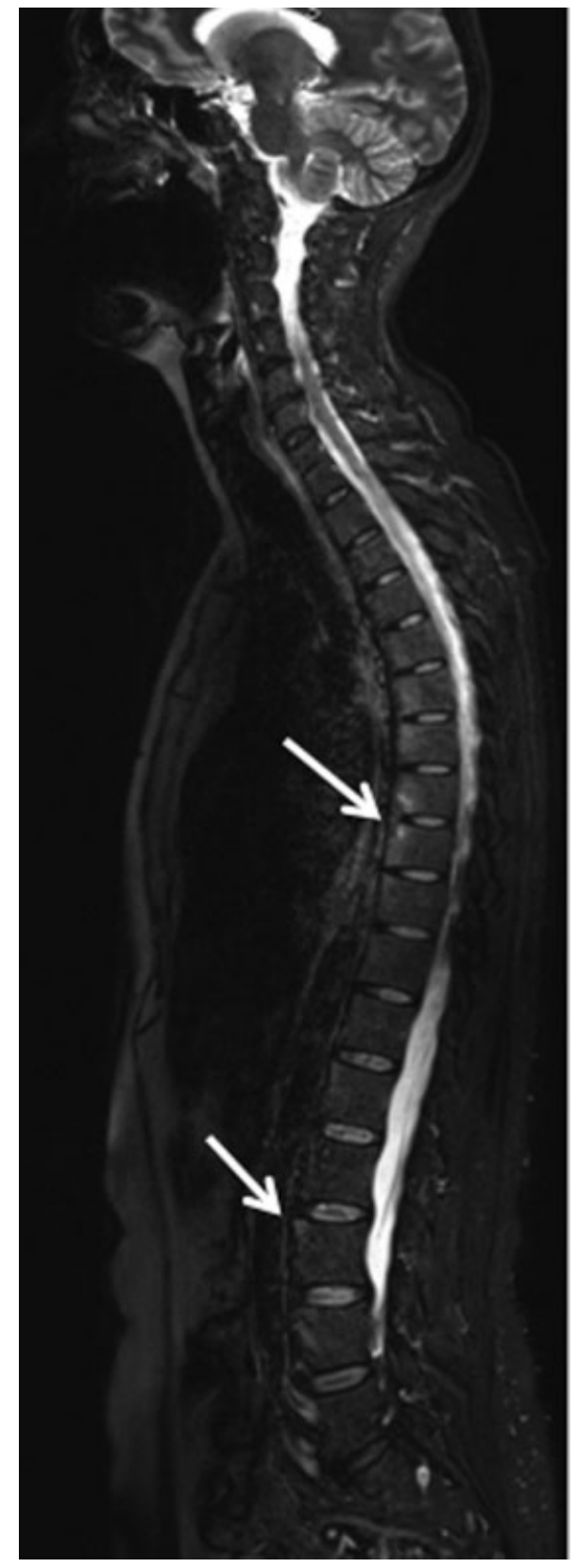

Fig. 12 Short tau inversion recovery sequence of the whole spine shows typical anterior corner inflammatory lesions in the thoracic and lumbar spine (arrows). These lesions are highly suggestive of spondylarthritis in this 32-year-old patient. 


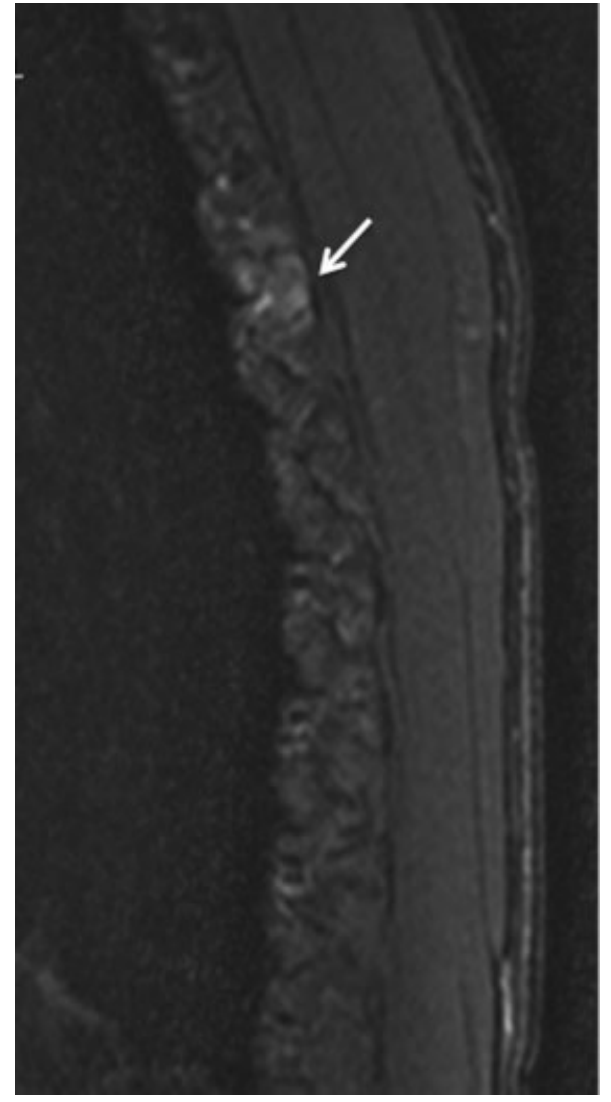

Fig. 13 Short tau inversion recovery sequence in this 20-year-old male patient shows bone marrow edema in the right costotransversal joint of the T5 vertebra (arrow). This location is typical for inflammatory involvement in spondylarthritis.

also be detected on radiographs and results in stiffening of the spine (-Fig. 11a, b).

\section{MRI}

A "positive" MRI for the spine is defined by the Outcome Measures in Rheumatology Clinical Trials (OMERACT) and
ASAS group if at least three anterior and/or posterior corner inflammation lesions (anterior or posterior spondylitis) or fat deposits at the vertebral corners are present. ${ }^{23}$ If two or more corner inflammatory lesions are present, the positive likelihood ratio for patients with AS increases to $11.5 .^{24}$ If the lesions are of high signal intensity and if the patient is $<45$ years of age, the corner inflammation lesions are highly suggestive for SpA (- Fig. 12). Both active and chronic inflammatory corner lesions can be present in one patient at the same time. In the chronic stage, or under tumor necrosis factor (TNF)- $\alpha$ inhibitor therapy, inflammatory corner lesions may turn into fatty infiltration. ${ }^{25}$ If located in the thoracic or upper lumbar spine, involvement of posterior elements of the spine including the facet joints, the costovertebral, costotransversal joints, and the pedicles are characteristic features in SpA ( - Fig. 13). In the acute phase, BME in these anatomical locations can be detected, whereas in the chronic phase, fatty infiltration or even ankylosis of the small joints can be discriminated. Syndesmophytes are generally described on radiographs, but, if prominent, they are seen on MRI as well.

Noninfectious spondylodiskitis is characterized by endplate BME and erosion at the vertebral end plates. Because these changes are similar to activated osteochondrosis, it might be helpful to analyze the disk (which is usually not dehydrated or herniated in spondylitis), to look at the location (degeneration typically involves levels L4-S1), and search for other pathognomonic features, such as corner inflammatory lesions (-Fig. 14a, b). If trauma is excluded, edema in the spinous process or at the ligament insertions on the spinous processes underlines the diagnosis of SpA (-Fig. 15).

\section{Differential Diagnosis}

\section{Sacroiliitis}

Diagnosing inflammatory sacroiliitis on MRI is not always straightforward, and, in some cases, it can be challenging. In addition, several alternative diagnoses can be suggested based on a characteristic MRI appearance (-Table 8).
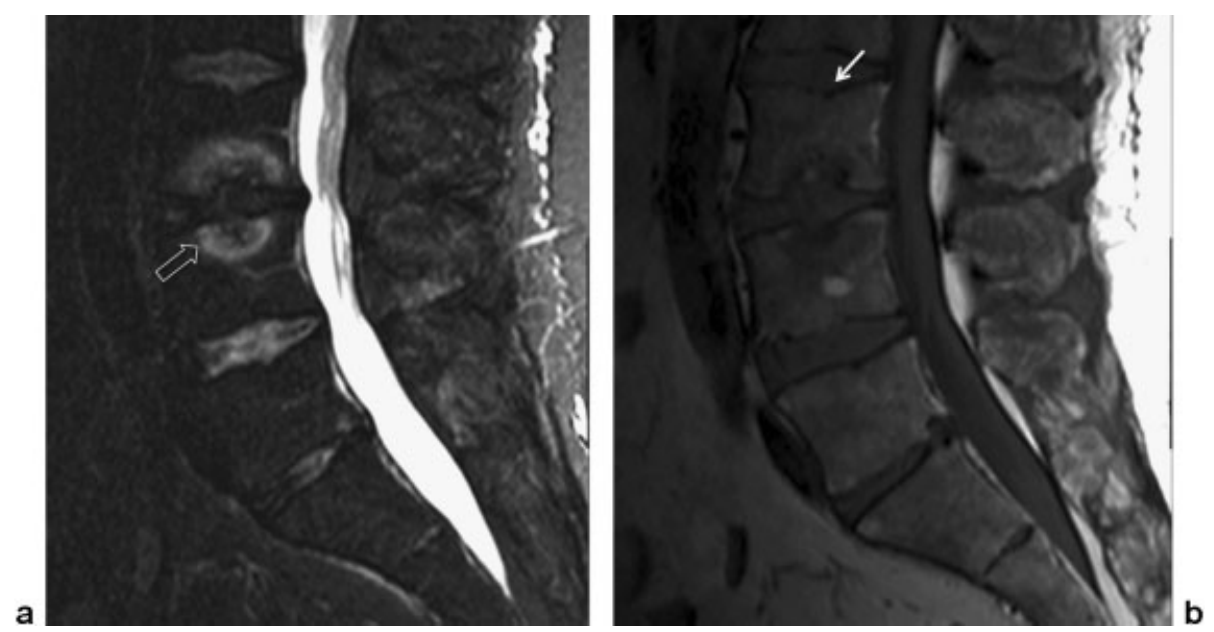

Fig. 14 (a) Subchondral bone marrow edema is detected by its high signal intensity on short tau inversion recovery at level L3-L4. (b) Erosions in the end plate are centrally located and nicely depicted on T1 (open arrow). Schmorl node should not be mistaken for an erosion (b, arrow). Obviously, in this case, there is an overlap between noninflammatory spondylodiskitis and activated osteochondrosis because the disk is dehydrated and slightly herniated. 


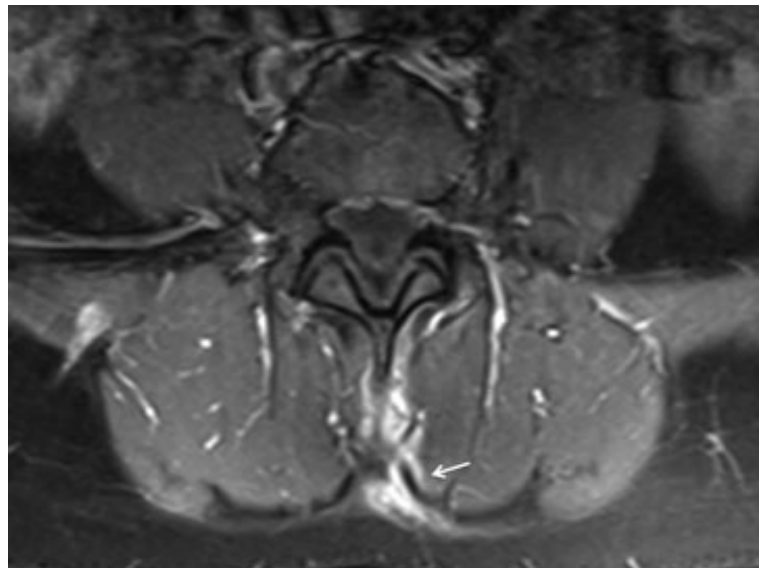

Fig. 15 Axial contrast-enhanced T1-weighted fat-suppressed sequence demonstrates the inflammation of the supraspinal ligaments (arrow).

\section{Infectious Sacroiliitis}

The MRI appearance of infectious sacroiliitis is the most similar to that of inflammatory sacroiliitis. In the acute phase, intense bone marrow edema is usually present on both sides of the SIJs, and excessive fluid and synovitis in the joint are seen. In more advanced cases, erosions and even bony bridges can be seen (-Fig. 16a, b). The major difference from inflammatory sacroiliitis is the crossing of anatomical borders, which is associated with involvement of the surrounding soft tissue, with intense edema and abscess formation of the iliac muscle in particular. ${ }^{26,27}$ After the acute phase of infection, erosions, fatty replacement, bony bridges, and even total joint ankylosis can be detected (-Fig. 17). Due to similarities in the MRI appearance, in subtle cases, only a precise clinical history can help to differentiate between the two entities.

\section{Osteitis Condensans Ilii}

Osteitis condensans ilii (synonym: hyperostosis triangularis ilii) is a condition thought to be related to remodeling of bone following stress across the SIJ, mostly seen in childbearing women with bone marrow edema in the perinatal period. Characteristically, sharply circumscribed triangular-shaped subchondral sclerosis, without erosions or joint space widen- ing, is detected anteriorly in the iliac side of the joint (-Fig. 18a, b). Minor bony irregularities or involvement of the sacral side are rare. Careful consideration should then be given to the clinical parameters and the characteristic location in the anterior part of the joint in order not to confuse this entity with inflammatory sacroiliitis.

\section{Insufficiency Fracture/Stress Reaction}

Stress reaction of the sacrum, not uncommon in recreational athletes, can be clinically confused with inflammatory sacroiliitis. The MRI appearance is quite different. Indeed, BME can be detected in the sacrum; however, it is usually located close to the sacral neuroforamen. In the later stage, a vertically oriented stress fracture can be detected as a hypointense irregular line that is surrounded by BME and spares the SIJs (-Fig. 19a, b). Because no excessive synovial fluid or structural lesions of the SIJs are present, this overuse syndrome can easily be differentiated from sacroiliitis.

\section{Osteoarthritis}

The MRI appearance of degenerative changes of the SIJ is similar to the degenerative findings seen in other joints. They include some joint irregularity with minimal subchondral sclerosis and subchondral cysts (-Fig. 20a, b) ${ }^{28}$ Fatty infiltration is not a characteristic finding, and usually, if BME appears, it is very subtle and confined to the immediate subchondral area at the iliac and sacral sites.

\section{Diffuse Idiopathic Skeletal Hyperostosis}

Anterior bridging osteophytes at the area of the SIJ anterior capsule characterize diffuse idiopathic skeletal hyperostosis (-Fig. 21a, b). ${ }^{27}$ Bridging osteophytes can also occasionally be seen posterior to the capsule, potentially leading to confusion with end-stage inflammatory sacroiliitis or degenerative changes in osteoarthritis.

\section{Anatomical Variants}

Several anatomical variants can mimic sacroiliitis. Accessory facets are quite common and appear either unilaterally or bilaterally in the posterior part of the joint. These accessory facets can lead to degenerative changes including BME and inflammation in the accessory joint area (-Fig. 22). However,

Table 8 Similarities and differences in the MR appearance of common alternative diagnoses to inflammatory sacroiliitis

\begin{tabular}{|c|c|c|c|c|c|}
\hline \multicolumn{6}{|c|}{ MRI findings } \\
\hline Diagnosis & BME & Erosions & Sclerosis & Fatty replacement & Bony bridge \\
\hline Septic sacroiliitis & + & $+1-$ & $+1-$ & $+1-$ & $+1-$ \\
\hline $\mathrm{OCl}$ & $+1-$ & - & + & - & - \\
\hline Stress reaction & + & - & - & - & - \\
\hline Osteoarthritis & $+1-$ & $+1-$ & $+1-$ & $+1-$ & - \\
\hline $\mathrm{DISH}$ & - & - & - & - & + \\
\hline Anatomical variants & $+1-$ & - & - & - & $+1-$ \\
\hline
\end{tabular}

Abbreviations: BME, bone marrow edema; DISH, diffuse idiopathic skeletal hyperostosis; OCI, osteitis condensans ilii. 

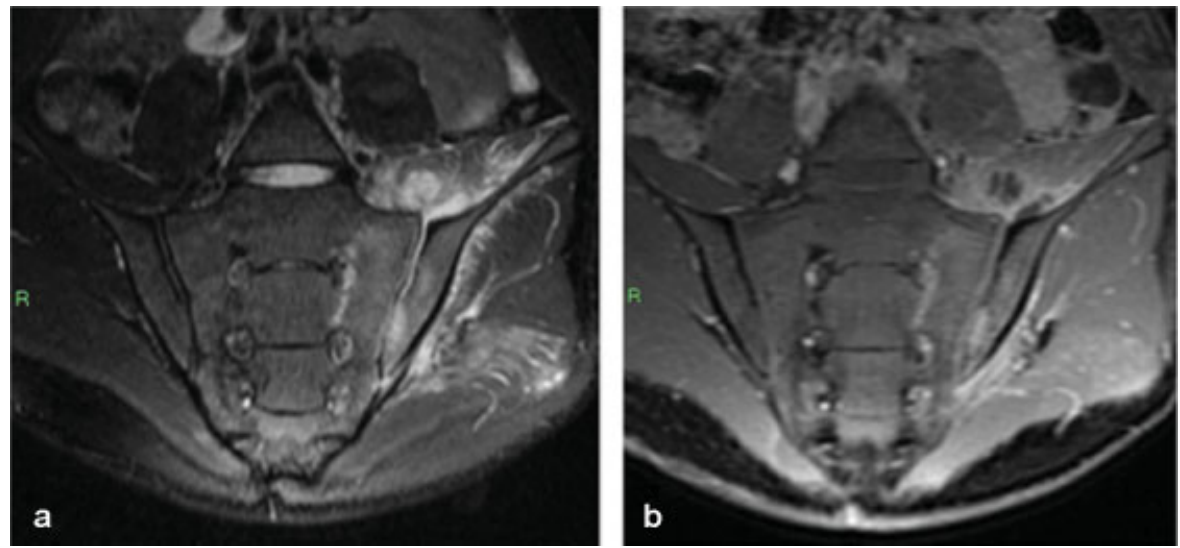

Fig. 16 Left septic sacroiliitis in a 19-year-old man. (a) Semicoronal short tau inversion recovery sequence shows bone marrow edema of the left sacroiliac joint, as well as excessive fluid in the joint. Intense soft tissue edema is noted anterior and posterior to the joint. (b) T1-weighted image after gadolinium injection with a fat suppression sequence. Abscess formation is detected anterior to the joint.

this location is posterior to the anatomical confinement of the SIJ and should not be confused with sacroiliitis.

Hemisacralization of the L5 vertebra to the sacrum can also lead to degenerative findings in this area, with BME and inflammation that is caused by the pseudarthrosis between the sacrum and the transverse process.

\section{Spinal Inflammation}

There is an overlap between degeneration and inflammation in the spine with regard to clinical symptoms and morphology on MRI. However, knowledge of certain morphological and clinical features facilitates the differentiation between the two entities. Other differential diagnoses, such as infection, tumor, and stress reaction/fractures, should be carefully excluded.

\section{Osteochondrosis MODIC Type I}

The most important differential diagnosis for noninfectious spondylodiskitis is activated osteochondrosis, which is sometimes impossible to distinguish ( $\mathbf{- F i g}$. 23a, b). In most cases,

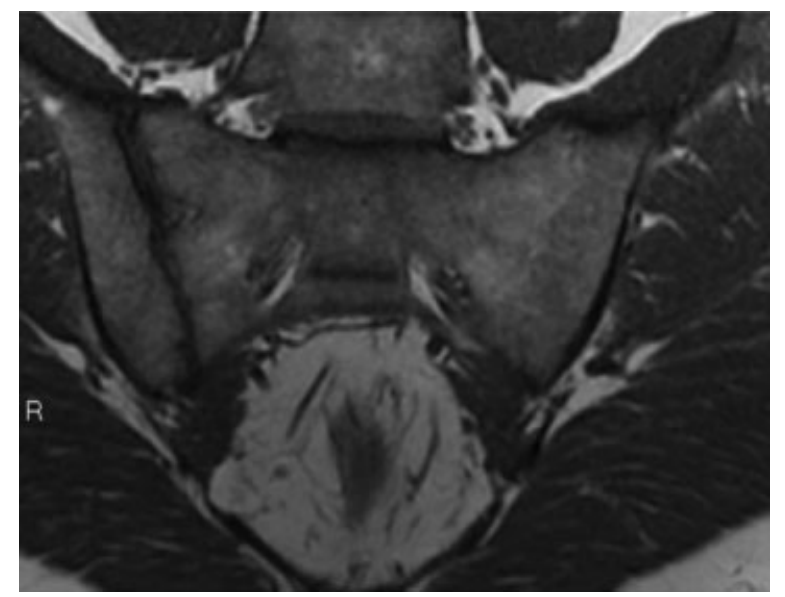

Fig. 17 Semicoronal T1-weighted image of the sacroiliac joint (SIJ) in a 30-year-old man with a history of septic sacroiliitis for 10 years before the MRI. Complete ankylosis of the left SIJ is demonstrated. The right SIJ is intact. it is helpful to look at the vertebral disk. A reduced height or herniation of the disk with low signal intensity on STIR or T2weighted images is more common in degeneration. Subchondrally located edema in the end plate is seen in both inflammation and osteochondrosis, with Modic type I changes. The distribution pattern, history, and age of the patient are helpful in differentiating the two entities. Levels L4-S1 are most commonly affected in degeneration.

\section{Infectious Spondylodiskitis}

Subchondral or extensive BME in the vertebral body might be caused by infection. High T2-weighted signal in the disk (fluid in disk sign) indicates diskitis, with increased contrast media uptake. Detection of paravertebral soft tissue inflammation or epidural abscesses enables the diagnosis of infectious spondylodiskitis (-Fig. 24a-c).

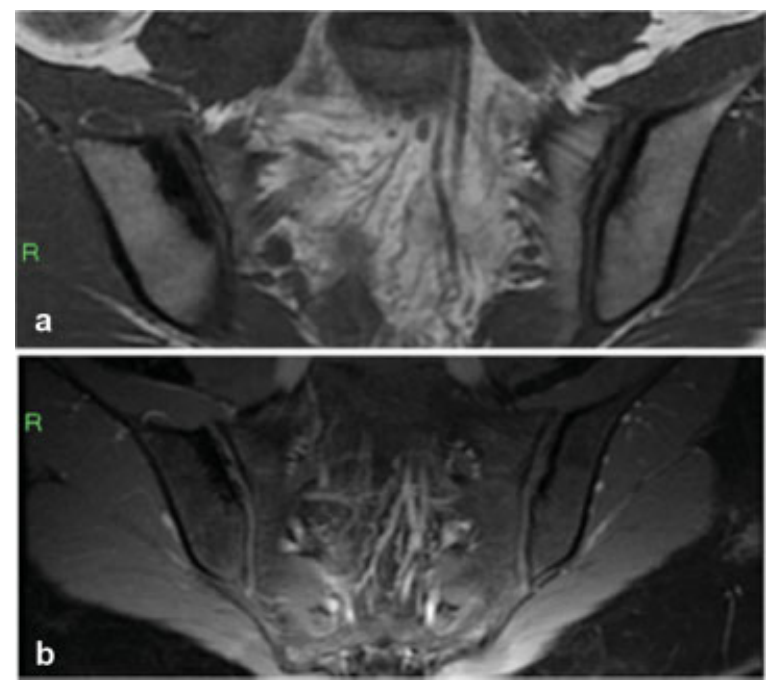

Fig. 18 Osteitis condensans ilii in a 34-year-old woman. (a) Semicoronal T1 and (b) short tau inversion recovery sequences demonstrate subchondral sclerosis of the anterior iliac part of the sacroiliac joint mainly on the right. Some minor bony irregularity but no overt erosions or bone marrow edema can be detected. 


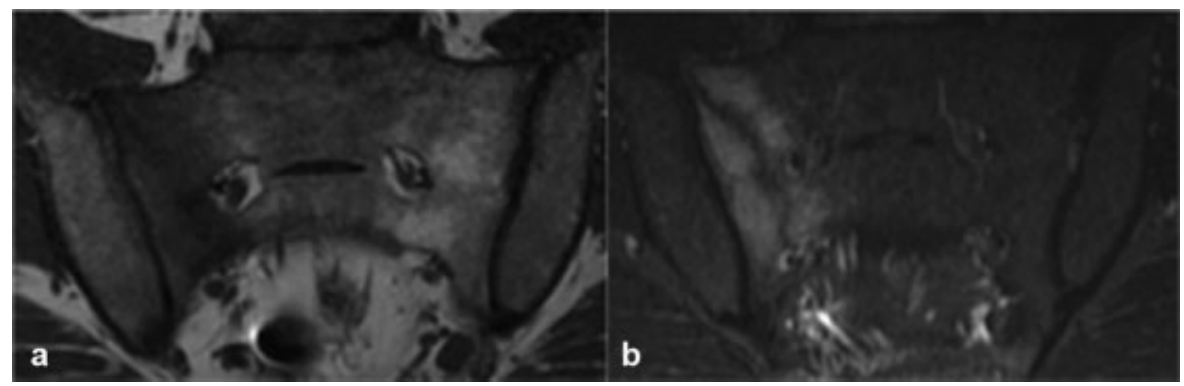

Fig. 19 (a) Semicoronal T1 and (b) short tau inversion recovery sequences of a 32-year-old recreational marathon runner show intense bone marrow edema of the sacral side of the right sacroiliac joint, with a vertical fracture line compatible with stress fracture.

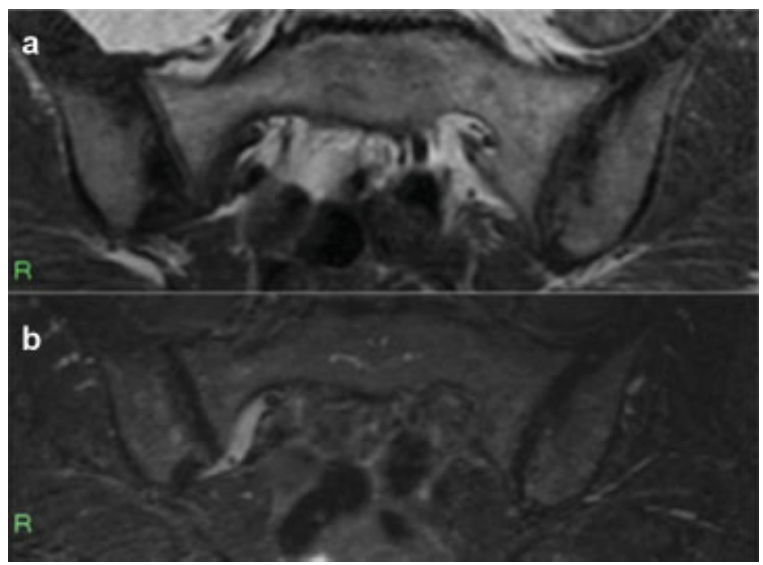

Fig. 20 (a) Semicoronal T1 and (b) short tau inversion recovery sequences of a 60-year-old women with psoriatic arthritis and suspected sacroiliitis. Very minor bone irregularity, subchondral sclerosis, and bone marrow edema can be detected mainly on the iliac side of the right and left sacroiliac joint. These are typical degenerative changes and should not be confused with structural and active inflammatory sacroiliitis changes.

\section{Spondylolysis/Stress Reaction}

Although inflammation in the lateral spinal segment has been described as pathognomonic, ${ }^{23}$ BME should be carefully evaluated and interpreted. Stress reaction in the pedicle or in later-stage spondylolysis can mimic inflammation. Athletes

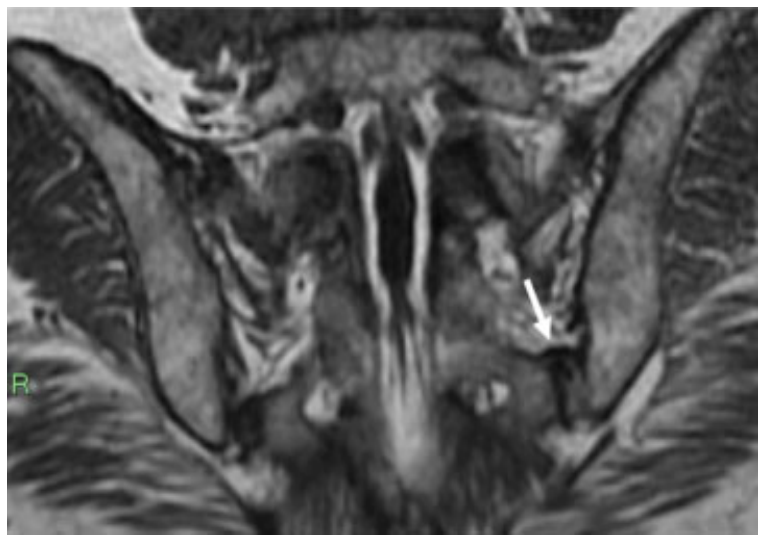

Fig. 22 Semicoronal T1-weighted images of a 25-year-old woman with an accessory facet posterior to the left sacroiliac joint (SIJ) (arrow). The SIJs on both sides are otherwise intact.

and patients with scoliosis and consecutive unilateral increased load on the facet joint are prone to experience stress reaction of the pedicles ( - Fig. 25a-c).

\section{Tumor}

Low signal intensity on T1-weighted images in the vertebral body might be due to edema or sclerosis. However,
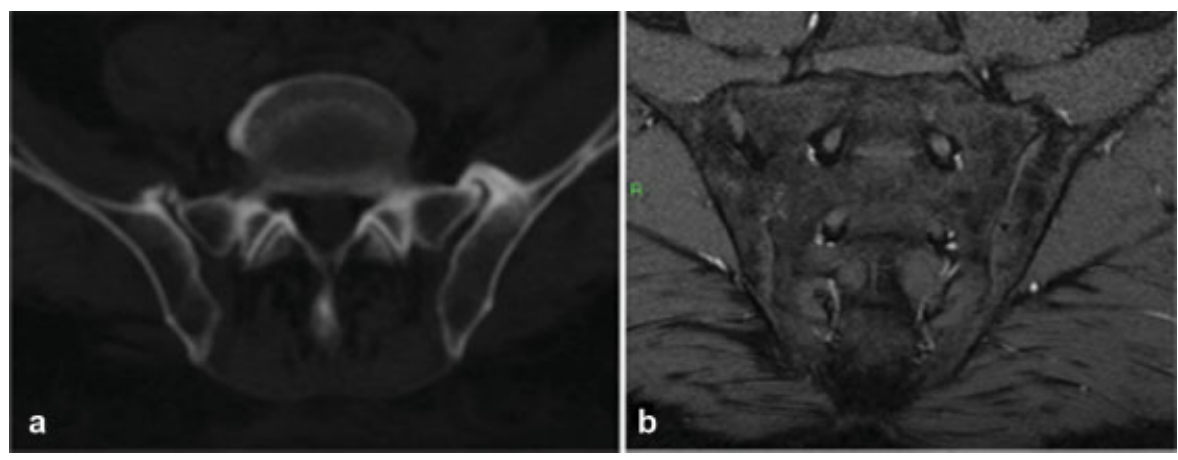

Fig. 21 (a) Axial computed tomography slice and (b) semicoronal short tau inversion recovery sequence of two different male patients with diffuse idiopathic skeletal hyperostosis, one 54 years of age (a) and the other 57 years of age (b). In both images, coarse bridging osteophytes can be noted anterior to the sacroiliac joint (SIJ). On the MRI, discrete bridging can also be noticed within the right SIJ joint itself. 

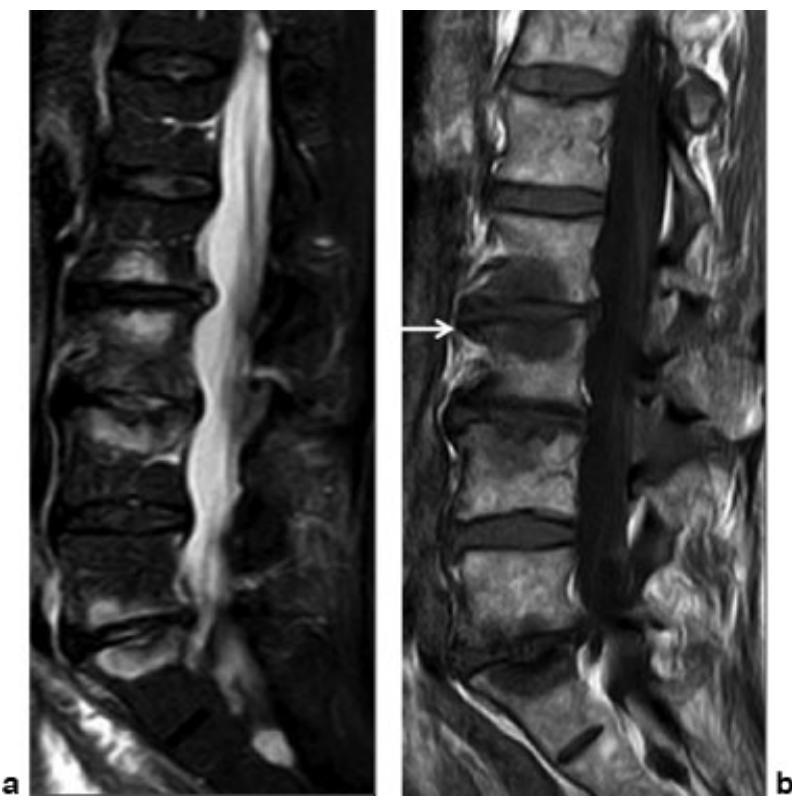

Fig. $23(\mathbf{a}, \mathbf{b})$ Subchondral bone marrow edema is seen on short tau inversion recovery (a) at levels $\mathrm{L} 2-\mathrm{L} 4$ and $\mathrm{L} 5-\mathrm{S} 1$. Note the reduced height of the intervertebral space and the herniation of the degenerated disk. The horizontal angulation of the osteophytes is typical for degeneration (arrow). displacement of fatty bone marrow caused by high numbers of (tumor) cells is of low signal intensity on T1-weighted sequences as well. Increased volume of the vertebral body, loss of concavity of the vertebral body borders, and destruction of the cortex with pathologic fracture are typical features of tumors. Patient history is crucial for interpretation of postradiotherapeutic changes of the bone marrow (-Fig. 26a-c).

\section{MR Artifacts}

\section{Wraparound Artifact}

A wraparound artifact occurs when the FOV is smaller than the body part being imaged. The aliasing can be compensated for by enlarging the FOV and by using presaturation bands on areas outside the FOV, or switching the phase and frequency directions, or by using surface coils.

\section{Coil Artifacts}

This artifact is caused by field inhomogeneities of the magnet and results in decreased fat suppression of the subcutaneous fat (-Fig. 27).

\section{Motion Artifacts}

Motion artifacts in the pelvis can be caused by peristalsis of the bowel or vessel pulsations, and can complicate the analysis of the SIJ. Motion artifacts are only seen in the phase-encoding direction, and changing the phase direction enables adequate image interpretation (-Fig. 28).
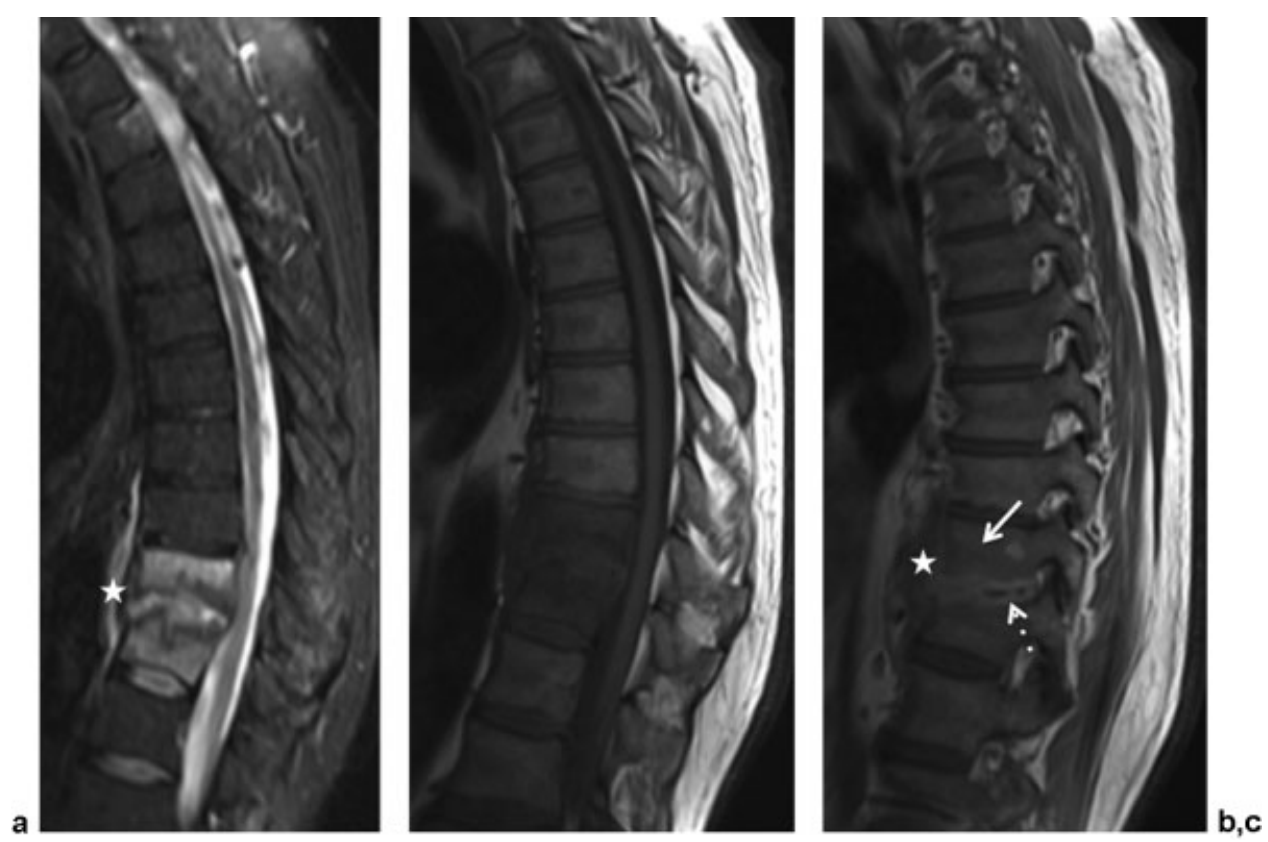

Fig. 24 Short tau inversion recovery sequence (a) shows extensive bone marrow edema in T10-11 vertebrae, with low signal intensity on the T1weighted sequence (b) and moderate contrast uptake (arrows) on the postcontrast T1-weighted image (c). The typical sign for spondylodiskitis is the fluid in disk sign, as well as the contrast uptake of the disk and the destruction of the end plate (dashed arrow). A prevertebral abscess is also seen (asterisks). 

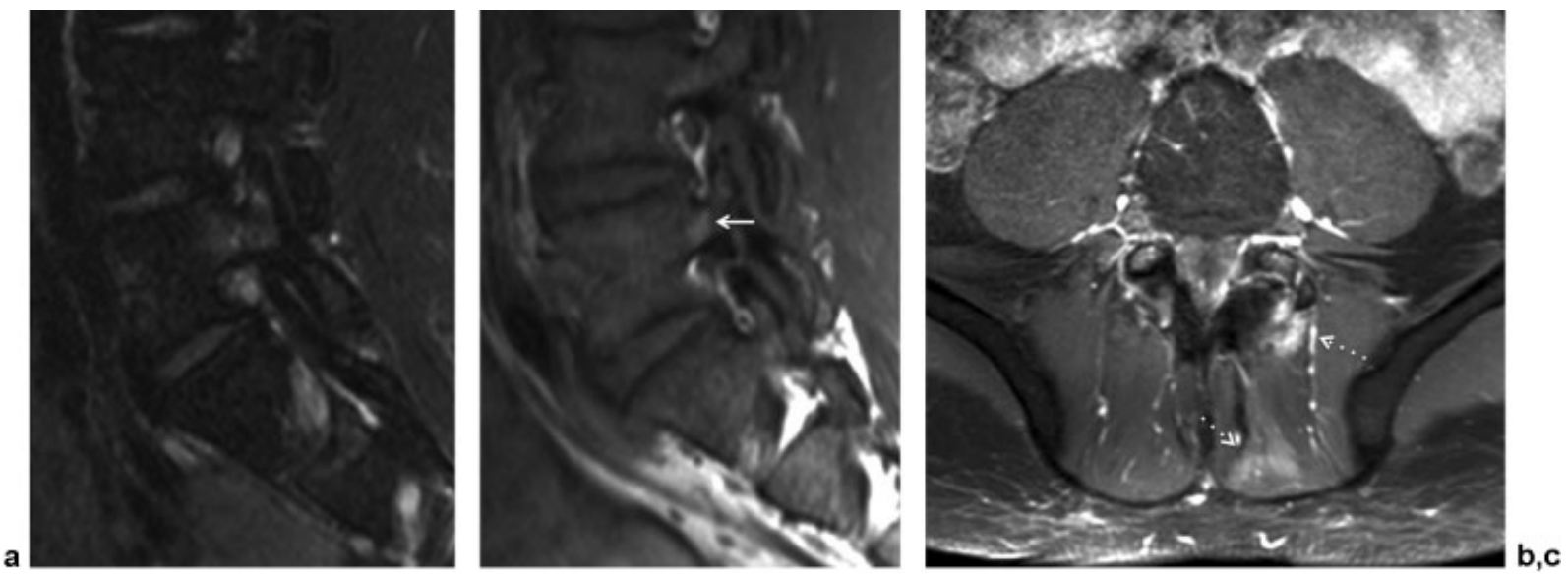

Fig. 25 (a-c) Short tau inversion recovery sequence (a) shows edema in the left pedicle with minor fat infiltration corresponding to high signal intensity on T1-weighted sequences (arrow) in a 24-year-old tennis player. Axial post-contrast-enhanced sequence shows the hypervascularization of the left facet joint and some edema in the left erector spinae and transversospinales muscles (dashed arrows). These findings were consistent with the diagnosis of overuse syndrome of the left L5-S1 facet joint and chronic stress reaction in the left pedicle.
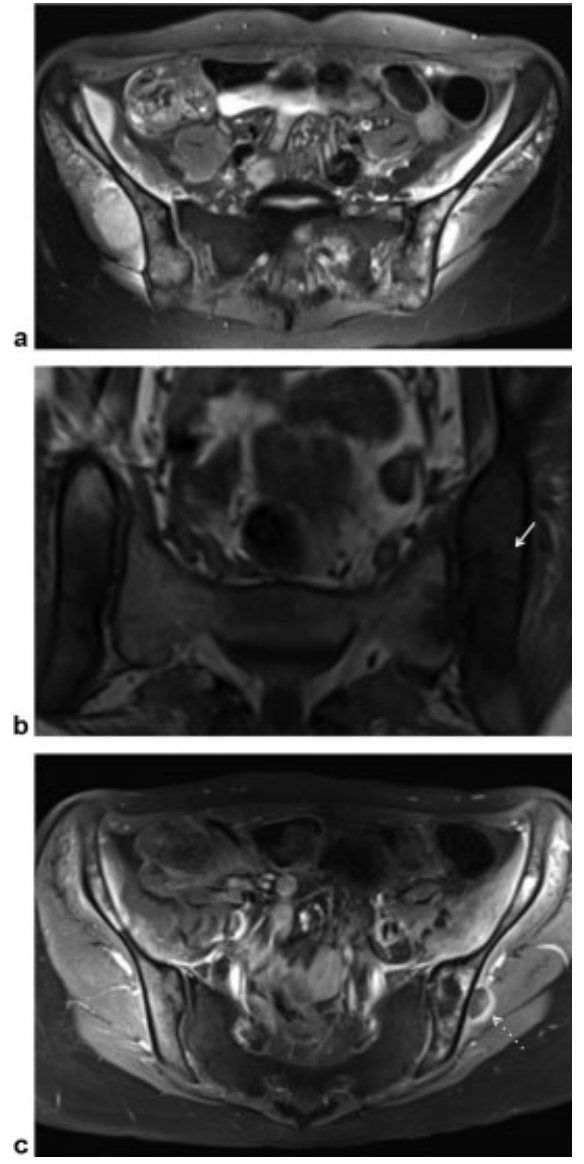

Fig. 26 (a) Extensive bone marrow edema in the pelvis is seen on short tau inversion recovery in this 21-year-old female patient with a Ewing sarcoma. (b) The fatty bone marrow is replaced and of low signal intensity on T1 (arrow). (c) Tumor masses infiltrate the surrounding muscle and show centrally necrotic areas on contrast-enhanced fatsuppressed T1 (dashed arrow).

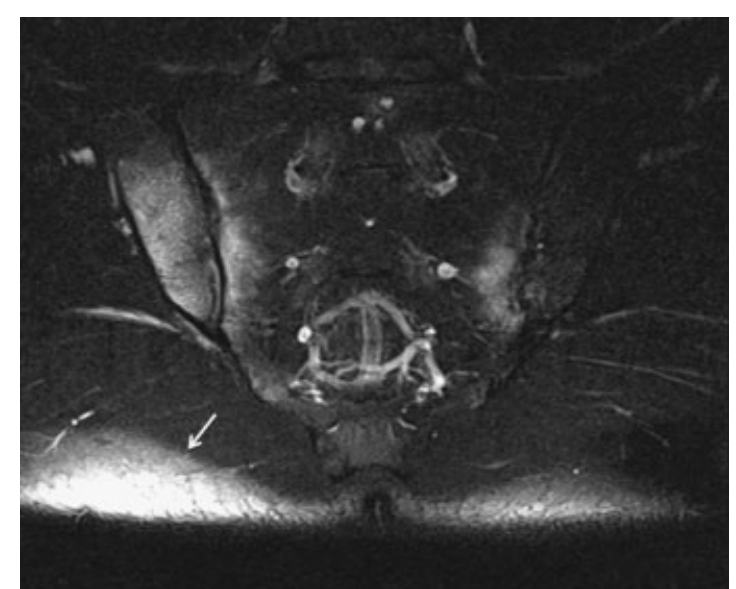

Fig. 27 Coil artifacts cause field inhomogeneities, which result in the reduced fat suppression in the subcutaneous fat tissue, primarily on the right side (arrow).

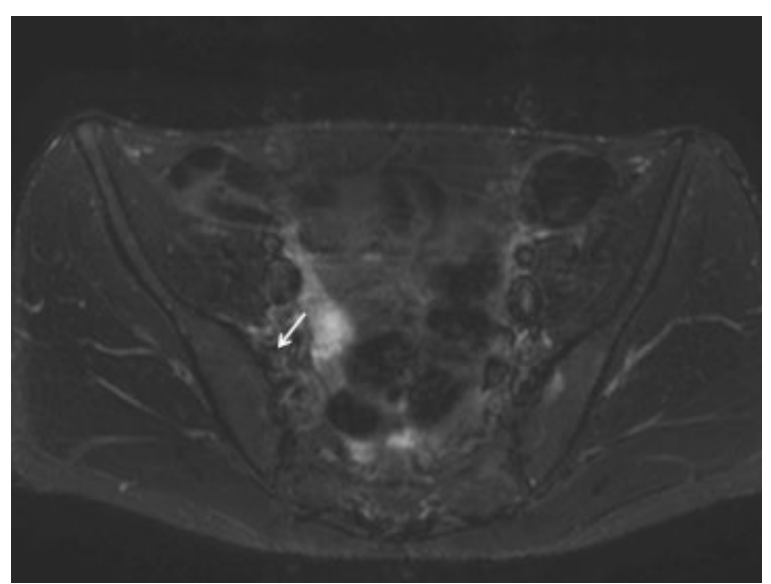

Fig. 28 Motion artifacts caused by the peristalsis of the bowel. It is impossible to differentiate between edemas of the sacroiliac joint and artifacts (arrow). Altering the phase-encoding direction avoids these artifacts. 


\section{Conclusions}

To improve patient outcomes in SpA, early diagnosis and early treatment are crucial prognostic factors. Because early inflammation is missed by radiography, MRI plays an important role in the diagnosis of early SpA. This consensus paper of the Arthritis Subcommittee of the European Society of Skeletal Radiology recommends MRI protocols of the spine and SIJs for 1.5- and 3-T MRI. A thorough knowledge of anatomy, morphologic SpA features in MRI and radiography, and differential diagnosis enables appropriate image interpretation.

\section{Acknowledgments}

We thank Mary McAllister for her thorough reading and editing of the manuscript.

\section{Conflict of interest}

The authors have nothing to disclose regarding the content of this article.

\section{References}

1 Barkham N, Keen HI, Coates LC, et al. Clinical and imaging efficacy of infliximab in HLA-B27-Positive patients with magnetic resonance imaging-determined early sacroiliitis. Arthritis Rheum 2009;60(4):946-954

2 Calin A, Porta J, Fries JF, Schurman DJ. Clinical history as a screening test for ankylosing spondylitis. JAMA 1977;237(24):2613-2614

3 Braun J, Sieper J. Ankylosing spondylitis. Lancet 2007;369(9570): 1379-1390

4 Sieper J, Rudwaleit M, Baraliakos X, et al. The Assessment of SpondyloArthritis international Society (ASAS) handbook: a guide to assess spondyloarthritis. Ann Rheum Dis 2009;68(Suppl 2): ii1-ii44

5 Said-Nahal R, Miceli-Richard C, Berthelot JM, et al. The familial form of spondylarthropathy: a clinical study of 115 multiplex families. Groupe Français d'Etude Génétique des Spondylarthropathies. Arthritis Rheum 2000;43(6):1356-1365

6 Rudwaleit M, Haibel H, Baraliakos X, et al. The early disease stage in axial spondylarthritis: results from the German Spondyloarthritis Inception Cohort. Arthritis Rheum 2009;60(3):717-727

7 Luong AA, Salonen DC. Imaging of the seronegative spondyloarthropathies. Curr Rheumatol Rep 2000;2(4):288-296

8 van der Linden S, Valkenburg HA, Cats A. Evaluation of diagnostic criteria for ankylosing spondylitis. A proposal for modification of the New York criteria. Arthritis Rheum 1984;27(4):361-368

9 Bennett AN, McGonagle D, O'Connor P, et al. Severity of baseline magnetic resonance imaging-evident sacroiliitis and HLA-B27 status in early inflammatory back pain predict radiographically evident ankylosing spondylitis at eight years. Arthritis Rheum 2008;58(11):3413-3418

10 Weber U, Lambert RG, Pedersen SJ, Hodler J, Østergaard M, Maksymowych WP. Assessment of structural lesions in sacroiliac joints enhances diagnostic utility of magnetic resonance imaging in early spondylarthritis. Arthritis Care Res (Hoboken) 2010; 62(12):1763-1771

11 Weber U, Pfirrmann CW, Kissling RO, Mackenzie CR, Khan MA. Early spondyloarthritis in an HLA-B27-positive monozygotic twin pair: a highly concordant onset, sites of involvement, and disease course. J Rheumatol 2008;35(7):1464-1466
12 Weber U, Lambert RG, Østergaard M, Hodler J, Pedersen SJ, Maksymowych WP. The diagnostic utility of magnetic resonance imaging in spondylarthritis: an international multicenter evaluation of one hundred eighty-seven subjects. Arthritis Rheum 2010; 62(10):3048-3058

13 Weber U, Zubler V, Zhao Z, et al. Does spinal MRI add incremental diagnostic value to MRI of the sacroiliac joints alone in patients with non-radiographic axial spondyloarthritis? Ann Rheum Dis 2014

14 Maksymowych WP, Chiowchanwisawakit P, Clare T, Pedersen SJ, Østergaard M, Lambert RG. Inflammatory lesions of the spine on magnetic resonance imaging predict the development of new syndesmophytes in ankylosing spondylitis: evidence of a relationship between inflammation and new bone formation. Arthritis Rheum 2009;60(1):93-102

15 Klauser AS, De Zordo T, Bellmann-Weiler R, et al. Feasibility of second-generation ultrasound contrast media in the detection of active sacroiliitis. Arthritis Rheum 2009;61(7):909-916

16 Baraliakos X, Davis J, Tsuji W, Braun J. Magnetic resonance imaging examinations of the spine in patients with ankylosing spondylitis before and after therapy with the tumor necrosis factor alpha receptor fusion protein etanercept. Arthritis Rheum 2005;52(4): 1216-1223

17 Puhakka KB, Melsen F, Jurik AG, Boel LW, Vesterby A, Egund N. MR imaging of the normal sacroiliac joint with correlation to histology. Skeletal Radiol 2004;33(1):15-28

18 Hermann KG, Landewé RB, Braun J, van der Heijde DM. Magnetic resonance imaging of inflammatory lesions in the spine in ankylosing spondylitis clinical trials: is paramagnetic contrast medium necessary? J Rheumatol 2005;32(10):2056-2060

19 Tse SM, Laxer RM. New advances in juvenile spondyloarthritis. Nat Rev Rheumatol 2012;8(5):269-279

20 Larbi A, Viala P, Molinari N, et al. Assessment of MRI abnormalities of the sacroiliac joints and their ability to predict axial spondyloarthritis: a retrospective pilot study on 110 patients. Skeletal Radiol 2014;43(3):351-358

21 Rudwaleit M, Jurik AG, Hermann KG, et al. Defining active sacroiliitis on magnetic resonance imaging (MRI) for classification of axial spondyloarthritis: a consensual approach by the ASAS/ OMERACT MRI group. Ann Rheum Dis 2009;68(10):1520-1527

22 Battistone MJ, Manaster BJ, Reda DJ, Clegg DO. Radiographic diagnosis of sacroiliitis-are sacroiliac views really better? J Rheumatol 1998;25(12):2395-2401

23 Hermann KG, Baraliakos X, van der Heijde DM, et al; Assessment in SpondyloArthritis international Society (ASAS). Descriptions of spinal MRI lesions and definition of a positive MRI of the spine in axial spondyloarthritis: a consensual approach by the ASAS/ OMERACT MRI study group. Ann Rheum Dis 2012;71(8): $1278-1288$

24 Weber U, Hodler J, Kubik RA, et al. Sensitivity and specificity of spinal inflammatory lesions assessed by whole-body magnetic resonance imaging in patients with ankylosing spondylitis or recent-onset inflammatory back pain. Arthritis Rheum 2009; 61(7):900-908

25 Song IH, Hermann KG, Haibel H, et al. Relationship between active inflammatory lesions in the spine and sacroiliac joints and new development of chronic lesions on whole-body MRI in early axial spondyloarthritis: results of the ESTHER trial at week 48. Ann Rheum Dis 2011;70(7):1257-1263

26 Stürzenbecher A, Braun J, Paris S, Biedermann T, Hamm B, Bollow M. MR imaging of septic sacroiliitis. Skeletal Radiol 2000;29(8): 439-446

27 Jans L, Van Praet L, Elewaut D, et al. MRI of the SI joints commonly shows non-inflammatory disease in patients clinically suspected of sacroiliitis. Eur J Radiol 2014;83(1):179-184

28 Tuite MJ. Sacroiliac joint imaging. Semin Musculoskelet Radiol 2008;12(1):72-82 